\title{
VALVE-IN-VALVE TRANSCATHETER AORTIC VALVE REPLACEMENT FOR THE TREATMENT OF NATIVE AORTIC INSUFFICIENCY
}

\author{
Rajika Jindani $^{1}$, Taylor James ${ }^{1}$, Sotiris Stamou ${ }^{2}$, and Marcos Nores ${ }^{2}$ \\ ${ }^{1}$ University of Miami Miller School of Medicine \\ ${ }^{2}$ JFK Medical Center
}

June 28, 2020

\begin{abstract}
Valve-in-valve transcatheter aortic valve replacement (VIV TAVR) has been recently utilized as an alternative for prohibitive surgical risk patients with aortic insufficiency of the native valves. When using this approach, placing a single bioprosthetic valve could possibly leave the patient with persistent significant aortic insufficiency. Based on these circumstances, we developed an approach where we place a second TAVR valve within an already positioned valve to achieve an appropriate result. We present two cases of an elective VIV TAVR in a TAVR valve in cases of aortic insufficiency.
\end{abstract}

\section{INTRODUCTION}

Historically, transcatheter aortic valve replacement (TAVR) has been used to treat patients with aortic stenosis, deemed high risk for surgical aortic valve replacement ${ }^{1}$. Recently, indications for TAVR have been expanded to intermediate and low-risk patients, and the use of TAVR in patients with aortic insufficiency is now being explored ${ }^{2}$. In this report, we will present and discuss the methodology employed in two cases of severe, symptomatic aortic insufficiency that underwent TAVR as treatment of pathology after being deemed prohibitive risk by an experienced Heart Valve team. The use of a Balloon-expandable Edwards SAPIEN valve to stabilize the initial deployment of a self-expandable Medtronic Evolut value is described.

\section{TECHNIQUE}

Ultrasound and fluoroscopic guidance were used to gain access to the bilateral common femoral arteries using a micropuncture needle technique. A $6 \mathrm{Fr}$ long sheath was placed in the left femoral vein. In the right common femoral artery, 2 Perclose ProGlide closure device was deployed in a pre-close fashion. A 5 Fr balloon tipped temporary transvenous pacemaker was placed in the right ventricular apex and a 6 Fr pigtail catheter was placed at the base of the aortic valve. Aortography was performed to obtain an optimal angle for valve deployment. The aortic valve was crossed via the right common femoral arterial approach and a long Confida wire was placed in the left ventricular apex.

In both cases, an initial Medtronic Evolut valve was advanced over a Confida wire and utilized for the TAVR-procedure. The valve was deployed with the assistance of small dye injections through the pigtail catheter, so as to carefully choose the location for deployment. After the valve was deployed, we noticed the valve had migrated into the Left Ventricular Outflow Tract (LVOT), generating significant perivalvular leakage. At that point, a Balloon-expandable Edwards SAPIEN valve was chosen and placed within the initial Medtronic-Evolut valve, decreasing the degree of aortic insufficiency to trace amounts.

\section{Case 1}


A 68-year-old male with ischemic cardiomyopathy, status post left ventricular assist device (LVAD), presented with symptoms of progressive shortness of breath and fatigue due to moderate-to-severe aortic insufficiency. The patient was assessed and considered prohibitive risk for aortic valve replacement by the Heart Failure Team and referred for TAVR evaluation. A transthoracic echocardiogram was done, showing left ventricular dysfunction with an ejection fraction of $10 \%$ and severe aortic insufficiency concerning for closed-loop recirculation (Figure 1).

We chose a Medtronic $34 \mathrm{~mm}$ Evolut with the goal of properly oversizing the native annulus. Since the valve was unable to provide a sustainable fix to the existing insufficiency, the LVAD flow was regulated during the procedure to avoid displacement of the bioprosthetic valve into the left ventricle by the existing regurgitation. At that point, we decided to place a \#29 size Balloon-expandable Edwards SAPIEN 3, oversizing it by adding an extra 4cc to the nominal valve volume and deploying it as a VIV within the aforementioned Medtronic Evolut valve. The overexpansion of the SAPIEN 3 Valve made it possible to anchor both valves in a noncalcified annulus (Figure 2). Postoperatively, a transesophageal echocardiogram showed an improved left ventricular ejection fraction of $15 \%$, a mean pressure gradient of $2.2 \mathrm{mmHg}$, and a LVOT diameter of 2 $\mathrm{cm}$. There were no complications from the procedure and excellent hemostasis was obtained. Patient was discharged home postoperative day 3. On further follow up, the patient felt significantly better and was extremely pleased with the results.

\section{Case 2}

A 75-year-old female presented with a Porcelain Aorta and a long-standing history of severe, symptomatic aortic insufficiency, with a severely calcified aorta throughout, a calcium score of 5888 , and diffuse aneurysmal dilation of the ascending aorta. She was deemed inoperable mostly because of frailty, severe pulmonary hypertension, and the associated Porcelain Aorta, and therefore consider for a TAVR procedure. A transthoracic echocardiogram prior to surgery showed adequate left ventricular systolic function with an ejection fraction of $60 \%$, dilation of the left ventricle, severe aortic insufficiency, and severe pulmonary artery hypertension with right ventricular systolic pressure of $80 \mathrm{mmHg}$ (Figure 3). Her aortic area was $452 \mathrm{~mm}^{2}$. The patient had a successful percutaneous right transfemoral TAVR with Medtronic $29 \mathrm{~mm}$ Evolut Pro valve implanted, followed by an Edwards SAPIEN 3, \#26, -1 cc bioprosthetic valve deployment inside the first valve (Figure 4). At the end of this procedure, there was trace aortic insufficiency present and excellent hemostasis confirmed by transesophageal echocardiography.

\section{DISCUSSION}

The development of aortic insufficiency in patients with LVAD is a common phenomenon - approximately 25$30 \%$ of patients develop this complication within one year ${ }^{6}$. This is likely the result of distorted valve function and reversal of flow dynamics across the aortic valve as a result of the LVAD device ${ }^{6}$. The development of aortic insufficiency results in increased heart failure symptoms, as well as a decrease in the efficacy of LVAD devices ${ }^{5}$. The risks associated with surgery have led surgeons to attempt less invasive procedures to treat aortic insufficiency in this population. The challenges present with a single valve TAVR include large annular diameter which cannot be properly covered by the Medtronic valve alone, inadequate access to a stable valve landing zone, and the potential for accelerated degeneration of transcatheter valve leaflets due to elevated heart pressures ${ }^{1}$.

In addition, this method was successful in reversing hemodynamics and the first valve served as a landing zone since there was a lack of valve calcification for the expandable valves to latch onto. Thus, valve malpositioning has been reported in up to $33 \%$ of patients ${ }^{7}$. In one study, $17 \%$ of patients required a second valve implantation, as seen in both patients in this report ${ }^{8}$. Despite precise measurements for implantation of the valve, mispositioning occurred because of unforeseen factors. For this reason, the second valve is implanted to decrease the risks and create a more sustainable solution for the insufficiency. The benefits of using a Medtronic valve was for positioning in the perfect location, as well as having the option to reposition the valve, if needed. The Edwards valve was used as the second valve because the overexpansion capacity could push the Medtronic valve towards the annulus and anchor the Edwards valve in the right position at 
the same time.

In previous studies, newer bioprosthetic heart valves have higher success rates than older generation valves ${ }^{8}$. However, even with the newer generation bioprosthesis used in this report, both Medtronic valves underwent migration, requiring second device implantation. It should be noted that in both cases Medtronic valves were attempted first, followed by Edwards valves. A previous study showed that under- and over-sizing of Evolut valves specifically leads to increased risk of device malpositioning. It was speculated in this study that this was due to the flared design of the Evolut models, which increases the risk of migration in noncalcified valves ${ }^{7}$. In these cases, however, the Medtronic valve provided a landing zone in the ascending aorta while the Edwards SAPIEN valve in turn provided greater internal forces, which helped to sufficiently stop the inappropriate backflow across the valve because of the maximized radial forces. Both together yield a sustainable solution that can be used in the future in similar cases of aortic insufficiency.

Previous studies done on the VIV TAVR technique bring up the possibility of increased stroke risk because of the manipulation of the atheromatous aorta, the hemodynamic instability which occurs during a procedure, and the possibility of stasis and nonlaminar blood flow around the second implanted valve ${ }^{4}$. These risks will have to be evaluated in the current cases as time goes on. A larger group of VIV implantations must be done to truly ascertain what risk may be present.

We recognize that the VIV in TAVR approach poses an increase in the immediate financial costs for the procedure, since two prosthetic valves must be used instead of one. The possibility of treating a prohibitive surgical risk patient with this catheter-based therapy may provide long-term benefits of durability and stability that could outweigh the initial costs. Many of the challenges faced by a single valve TAVR are addressed and improved upon with a second valve. The findings of this case study indicate that using TAVR for treatment of aortic insufficiency is feasible; however, surgeons should plan for the likely indication for valve-in-valve implantation.

\section{STUDY LIMITATIONS}

Limitations of our study include the small sample size, as well as lack of long-term follow-up. Thus, the long-term consequences of this intervention cannot be commented on to firmly assert that this has been a success. Longitudinal studies assessing the efficacy of this VIV TAVR method with both Medtronic and Edwards valves are necessary to determine if there is a benefit in the overall quality of life and mortality.

\section{CONCLUSIONS}

In conclusion, in our series, a planned valve-in-valve TAVR in patients with aortic insufficiency, although not commonly used, is a reasonable option for patients who are unable to undergo an operative surgical aortic valve replacement. As the use of LVAD increases, symptomatic aortic insufficiency in this group of high-risk patients will become an increasingly common challenge. Further studies are needed to evaluate the efficacy of TAVR and VIV TAVR for the treatment of native or LVAD associated aortic insufficiency.

\section{Conflict of Interest}

None to report.

\section{Disclaimer}

This research was supported (in whole or in part) by HCA Healthcare and/or an HCA Healthcare affiliated entity. The views expressed in this publication represent those of the author(s) and do not necessarily represent the official views of HCA Healthcare or any of its affiliated entities.

\section{REFERENCES}

1. Kozarek, Katherine, et al. "Transcatheter Aortic Valve Replacement for Left Ventricular Assist DeviceInduced Aortic Insufficiency." Journal of Cardiothoracic and Vascular Anesthesia, vol. 32, no. 4, 2018, pp. 1982-1990., doi:10.1053/j.jvca.2018.01.013. 
2. D'ancona, Giuseppe, et al. "TAVI for Pure Aortic Valve Insufficiency in a Patient With a Left Ventricular Assist Device." The Annals of Thoracic Surgery , vol. 93, no. 4, 2012, doi:10.1016/j.athoracsur.2011.11.019.

3. Reul RM, Ramchandani MK, Reardon MJ. Transcatheter Aortic Valve-in-Valve Procedure in Patients with Bioprosthetic Structural Valve Deterioration. Methodist Debakey Cardiovasc J . 2017;13(3):132141. doi:10.14797/mdcj-13-3-132

4. Giri, Jay, et al. "Procedural and Clinical Outcomes of the Valve-in-Valve Technique for Severe Aortic Insufficiency after Balloon-Expandable Transcatheter Aortic Valve Replacement." Catheterization and Cardiovascular Interventions, vol. 80, no. 1, 2012, pp. 139-147., doi:10.1002/ccd.23451.

5. Pal, Jay D., et al. "Transcatheter Aortic Valve Repair for Management of Aortic Insufficiency in Patients Supported with Left Ventricular Assist Devices." Journal of Cardiac Surgery, vol. 31, no. 10, 2016, pp. 654-657., doi:10.1111/jocs.12814.

6. Fried JA, Nazif TM, Colombo PC. A new frontier for TAVR: Aortic insufficiency in CF-LVAD patients. The Journal of Heart and Lung Transplantation. 2019;38(9):927-929.

7. De Backer O, Pilgrim T, Simonato M, et al. Usefulness of Transcatheter Aortic Valve Implantation for Treatment of Pure Native Aortic Valve Regurgitation. The American Journal of Cardiology.2018;122(6):1028-1035.

8. Gafoor S, Sharma R. TAVR for Pure Native Aortic Regurgitation and Failing Regurgitant Surgical Bioprostheses: Alternative Indication or Alternative Fact?. JACC: Cardiovascular Interventions.2017;10(10):1057-1059.

\section{FIGURE LEGENDS}

Figure 1. Valve and degree of insufficiency before placement of bioprosthetic valves in Case 1.

Figure 2. Placement of Edwards SAPIEN 3, \#29, $+4 \mathrm{cc}$ bioprosthesis inside a Medtronic $34 \mathrm{~mm}$ Evolut PRO in Case 1.

Figure 3. Valve and degree of insufficiency before placement of bioprosthetic valves in Case 2. The perivalvular leakage is visible in this image.

Figure 4. Placement of an Edwards SAPIEN 3, \#26, -1 cc bioprosthetic inside a Medtronic 29 mm Evolut Pro valve in Case 2.

\section{Hosted file}

Case Report Figures.docx available at https://authorea.com/users/337427/articles/463029valve-in-valve-transcatheter-aortic-valve-replacement-for-the-treatment-of-nativeaortic-insufficiency 